\title{
Article
}

\section{Performance of diabetes screening tests: an evaluation study of Iranian diabetes screening program}

\author{
Fateme Kianpour 1, Mohammad Fararouei 2,*, Jafar Hassanzadeh 2, \\ Mohammadnabi Mohammadi ${ }^{3}$, Mostafa Dianatinasab 4 \\ ${ }^{1}$ Student research center, Department of Epidemiology, Shiraz University of Medical Sciences, Shiraz, Iran. \\ ${ }^{2}$ Department of Epidemiology, Shiraz University of Medical Sciences, Shiraz, Iran. \\ ${ }^{3}$ Health Deputy, Gerash University of Medical Sciences, Gerash, Iran. \\ ${ }^{4}$ Department of Epidemiology, Maastricht University, The Netherlands \\ * Correspondence:: Professor Mohammad Fararouei; Shiraz University of Medical Sciences, \\ Shiraz, Islamic Republic of Iran. Email: fararouei@sums.ac.ir; ORCID: https://orcid.org/0000- \\ 0001-9362-328X; Tel-Fax: 98-71-32701020
}

\begin{abstract}
Background: Diabetes is a common non-communicable disease that is responsible for about $9 \%$ of all deaths and $25 \%$ reduction in life expectancy and nearly half of the diabetic patients are not aware of their disease. In this regard; diabetes screening to identify un-known diabetic patients is of great importance. Aims: The aims of this study were first to evaluate the performance of two commonly used diabetes screening tests that are currently recommended by the Iranian national screening program for diabetes (NSPD). Methods: The validities of the two diabetes screening tests were measured among 1057 participants older than 30 years. The studied screening tests included Capillary fasting blood glucose (CBG) and glycated hemoglobin (HbA1c). The golden standard for measuring the validity of the tests was venous fasting plasma glucose (VPG). Results: According to the results; the sensitivity of CBG and HbA1c tests were $69.01 \%$ and $84.5 \%$ and the specificity of the tests were $95.7 \%$ and $79.3 \%$ respectively. Positive and negative predictive values were $53.84 \%$ and $97.72 \%$ for $\mathrm{CBG}$ and $22.72 \%$ and $98.61 \%$ for $\mathrm{HbA} 1 \mathrm{c}$ respectively. The recommended cut-points for CBG and $\mathrm{HbA1c}$ were $116.5 \mathrm{mg} / \mathrm{dl}$ and $7.15 \%$ respectively. Using these values as the new cut-points; sensitivity and specificity of CBG and $\mathrm{HbA} 1 \mathrm{c}$ changed to $80.30 \%$ and $89.10 \%$, and $77.50 \%$ and $94.20 \%$ respectively. Conclusions: Compared to several other countries; the performance of NSPD is relatively higher in Iran. ROC analysis suggested new cut-points for significantly better performance of NSPD.
\end{abstract}

Keywords: Diabetes mellitus; screening; HbA1c; fasting plasma glucose

\section{Background:}

Several factors including socio-economic development and significant progress in health and medical cares reduced mortality at a younger age and raised life expectancy. On the other hand, these changes along with the new sedentary lifestyles caused sharp rises in several chronic diseases. (1) Diabetes mellitus (DM) is a common metabolic disease (2) that about one-half of the patients are unaware of their condition (3). In addition, about $9 \%$ of total deaths and $25 \%$ reduction in life expectancy are directly or indirectly associated with diabetes $(4,5)$. For example, cardiovascular diseases are among the most common diabetes-related causes of deaths and about $43 \%$ of deaths due to diabetes occur among individuals under 70 years of age (6). This means that DM kills patients when they are still socio-economically active. (1). It is also estimated that about $12 \%$ of the global health budget is being spent on diabetes and its related conditions (7). Apart from the above facts, 
figures suggest that DM is alarmingly on rising and it is becoming a serious problem threatening global health and economy (8). Reports suggested that the global prevalence of DM among individuals over 18 years of age was about $9 \%$ in 2014. (9) However, the international diabetes federation (IDF) has estimated that the prevalence of diabetes is yet to be raised to $9.9 \%$ in 2030. (10) With regard to the geographical distribution of diabetes, it is estimated that $20 \%$ of the world's diabetic patients are living in South East Asia and it is predicted that in the near future, the Asian population is more seriously affected by DM compared to the population of the other parts of the world $(11,12)$.

As the epidemic of DM is expanding, the costs of the disease, including the cost of diagnosis and treatment of its complications are also rising sharply. It is because, on average, the cost of medical complications among DM patients is about 3.2 times higher than the costs of treating non-diabetics patients for the corresponding conditions (13). As a result, in addition to the severity and a wide range of health conditions, the economic damages caused by DM in various countries are remarkable. This is why the socio-economic burden of DM in low or middle-income countries affects their development (14). Similar to the rest of the world including the Middle East, in Iran over the past three decades the prevalence of diabetes has become doubled. Based on an estimate in 2013, the prevalence of DM in Iran was 13.8\% (12) and the incidence was about 1.6 per 100 person-year among individuals older than 20 years of age (15). It is also estimated that in 2014, 38079 deaths occurred among the Iranian population due to cardiovascular complications caused by DM (16). Reports suggested that in Iran a huge amount of money is being spent on the treatment of diabetes and its related health problems. In this regard, early DM detection is essential in the prevention and management of the severe and irreversible complications (17).

\section{The Iranian National Screening Program for Diabetes (NSPD):}

Due to the high cost of treatment, life-threatening complications, and relatively high prevalence, applying effective screening programs to identify people with undiagnosed DM is of utmost importance to the Iranian ministry of health. As a result, the Iranian ministry of health has recently implemented a routine diabetes screening program (DSP) into primary health care services. The program is to detect undiagnosed diabetes cases among the rural population who are older than 30 years of age. From 2016, DPS is being conducted by health centers and health houses to diagnose diabetes among the target population with at least one of the following risk factors: $\mathrm{BMI} \geq 30$, whist circumference $\geq 100$ for men (or $\geq 86 \mathrm{~cm}$ for women), family history of DM and history of gestational diabetes (among women). In that regard, all rural residences over 30 years of age are to be annually screened by the public health service providers (Figure1). Accordingly, the individuals are invited to the health houses to be visited by rural health nurses and voluntary health workers. The eligible individuals are asked to fast for at least 8 hours prior to the morning that they have an appointment to visit the health house that they are registered with. In the health house, the person's capillary fasting blood glucose (CBG) is measured with a glucometer. If the result of the CBG test was positive (FBS $\geq 126 \mathrm{mg} / \mathrm{dl}$ ), the individuals are referred to a health center to take a Venus plasma glucose (VPG) test (as a diagnostic test). A VPG test result equal to or bigger than $126 \mathrm{mg} / \mathrm{dl}$ is considered positive for DM.

\section{Aims:}

This study is conducted to evaluate the performance of Iranian DSP and its recommended cut points for the selected screening tests. In particular, this study aimed to evaluate sensitivity, specificity, and predictive values of CBG and HbA1c test, using fasting plasma glucose (VPG) as the gold standard (18). 


\section{Material and methods:}

The settings:

This study was conducted among rural residences of Gerash county, which is located in the southern part of Fars province, Iran. The county consists of 25 villages with about 14456 rural residents. In the study area, 7 health houses and 2 rural health centers deliver primary health services to the defined population.

Figure 1. presents the flowchart of the procedures of the Iranian DSP and the additional steps taken by the current study (rectangles).

Figure 1. Flowchart of the Iranian diabetes screening program and the current study (rectangles represent additional steps taken by currents.

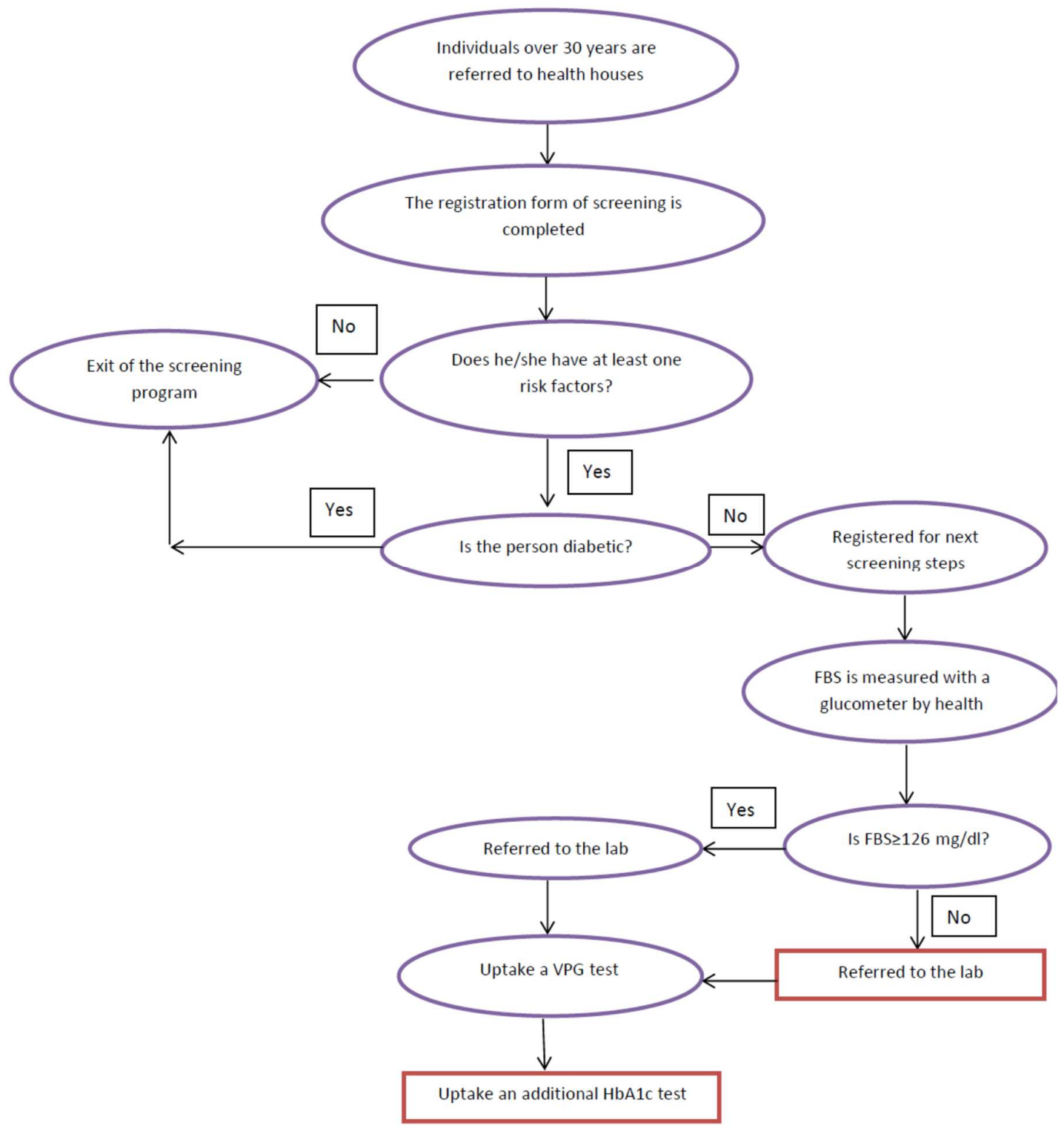

Data collection:

The present study recruited 1057 participants living in rural areas of the County. All participants were over 30 years of age and had at least one of the screening criteria defined by DSP (mentioned 
before). The participants were invited to the health houses and were interviewed by 7 experienced and trained health nurses. The required data was collected via an interview-administered questionnaire, which was specially designed and evaluated for DSP by the ministry of health. The questionnaire includes demographic data and a history of DM in the participants or their relatives. In addition, CBG was measured with a glucometer (Easy Gloco). The blood sample was taken from the toe of the middle finger of the left hand. After CBG test was conducted, irrespective of the result, the participants were referred to a public laboratory based in the nearby health center for conducting VPG test. The test was performed by a classic Alpha auto analyzer. In addition, at the same time, all participants gave a $\mathrm{HbA1C}$ test which was conducted by nicocard reader device using the glucose oxidase method. Like many other studies, which measured sensitivity and specificity of diabetes screening tests, this study followed WHO's recommendations suggesting VPG as the gold standard for evaluation capillary blood glucose and HbA1C tests (18).

Inclusion criteria: All participants were included providing they were over 30 years of age, had either of the above-mentioned risk factors defined by DSP, and reported no history of DM. Women were also to be not pregnant or breastfeeding. As many of the participants were illiterate, verbal informed consent was obtained from each participant before the interview. The study protocol is approved by Shiraz University of Medical Sciences research committee (approval number=94-01-0410908).

Sampling and statistical methods:

Sample size ( $\mathrm{n}=1010)$ was calculated based on the global prevalence of diabetes and using the formula provided by Karim Allah Hajian (19). In practice, however, all individuals over 30 years of age with one or more of the previously mentioned risk factors were recruited ( $\mathrm{n}=1057)$.

The collected data was analyzed in SPSS 19 using frequency, cross-tabs and chi-square test. In addition, R4.0 was used to conduct ROC analysis to define the best cut points for CBS and HbA1c in screening DM among the study population.

\section{Results:}

In total, 1057 individuals, who were over 30 and were living in rural areas of Gerash county participated in this study. The sex ratio (female/male) of the sample was $2(\mathrm{P}<0.05)$ and almost similar age distributions in the two genders were observed $(P>0.05)$. The frequency distribution of the participants based on their test results is presented in Table1. Accordingly, the rate of positive results of FBS test (setting $\geq 126 \mathrm{mg} / \mathrm{dl}$ as a cut point) based on CBG and VPG ( $8.60 \%$ and $6.70 \%$ respectively) were significantly different $(\mathrm{P}<0.001)$. However, according to the results of $\mathrm{HbA1c}$, the prevalence of DM was $25 \%$ when the cut point was set at $6.50 \%$ (as recommended by DSP) (20).

Table 1. Prevalence of DM based on the results of three diagnosis testes (CBG, VBG and HbA1c).

\begin{tabular}{cccc}
\hline variable & $\mathrm{N}$ & $\mathrm{N}^{\dagger}$ & $\%^{*}$ \\
& Total & Suspected DM & \\
\hline CBG & 1057 & 91 & 8.60 \\
\hline VBG & 1057 & 71 & 6.70 \\
\hline HbA1c & 1057 & 264 & 25 \\
\hline
\end{tabular}

†Based on WHO recommended cut points ( $\geq 126 \mathrm{mg} / \mathrm{dl}$ for CBG and VPG and $6.50 \%$ for HbA1c) 
The validity of $C B G$ and $H b A 1 c$ tests:

Using VPG $(\geq 126 \mathrm{mg} / \mathrm{dl})$ as the gold standard for diagnosis of DM, sensitivity, specificity, positive predictive values (PPV) and negative predictive values (NPV) for CBG and HbA1c are calculated and presented in table 2. As presented in Figure 2, receiver operating characteristic analysis provided new cut-points for the screening of diabetes based on CBG and HbA1c. Accordingly, at the DSP recommended cut-points, the areas under the curve (AUC) were $88.6 \%$ and 92.8\% for CBG and $\mathrm{HbA1c}$ respectively. However, the suggested new cut points obtained by ROC analysis for the screening tests seem to provide better performance among the study population (Table 3). Accordingly, the optimum cut points for CBG (116.50 mg/dl) and HbA1c (7.15\%) are considerably lower than those that were recommended by NSPD. Using these values as new cut points, sensitivity and specificity of CBG raised from $69.01 \%$ to $80.30 \%$ and decreased from $95.74 \%$ to $89.10 \%$ respectively. Similarly, using $7.15 \%$ as a cut point for $\mathrm{HbA} 1 \mathrm{c}$ test, sensitivity and specificity changed from $84.50 \%$ to $77.50 \%$ and from $79.31 \%$ to $94.20 \%$ respectively (Table 4 ).

Table 2. Sensitivity, specificity, PPV and NPV of CBG and HbA1c based on WHO's recommended cut points.

\begin{tabular}{|c|c|c|c|c|c|c|}
\hline Screening & Clinical & $\mathrm{N}$ & Sensitivity & Specificity & PPVIt & NPVItt \\
\hline Variable & reference $^{\dagger}$ & Total & & & & \\
\hline \multirow[t]{2}{*}{$\mathrm{CBG}^{*}$} & VBG† & 1057 & $49 / 71$ & $944 / 986$ & $49 / 91$ & $944 / 966$ \\
\hline & & & (69.01\%) & (95.74\%) & (53.84\%) & (97.72\%) \\
\hline \multirow[t]{2}{*}{$\mathrm{HbA}_{1} \mathrm{c}^{\wedge}$} & VBG $†$ & 1057 & $60 / 71$ & $782 / 986$ & $60 / 264$ & $782 / 793$ \\
\hline & & & $(84.50 \%)$ & (79.31\%) & $(22.72 \%)$ & $(98.61 \%)$ \\
\hline
\end{tabular}

\footnotetext{
†CBG $\geq 126 \mathrm{mg} / \mathrm{dl}$

†VPG $\geq 126 \mathrm{mg} / \mathrm{dl}$ and clinical diagnosis

††PPV=Positive predictive value

$\Pi+\mathrm{NPV}=$ Negative predictive value

${ }^{\wedge} \mathrm{HbA} 1 \mathrm{c} \geq 6.5 \%$
} 
Figure 2: ROC curve with screening variables and VPG as reference

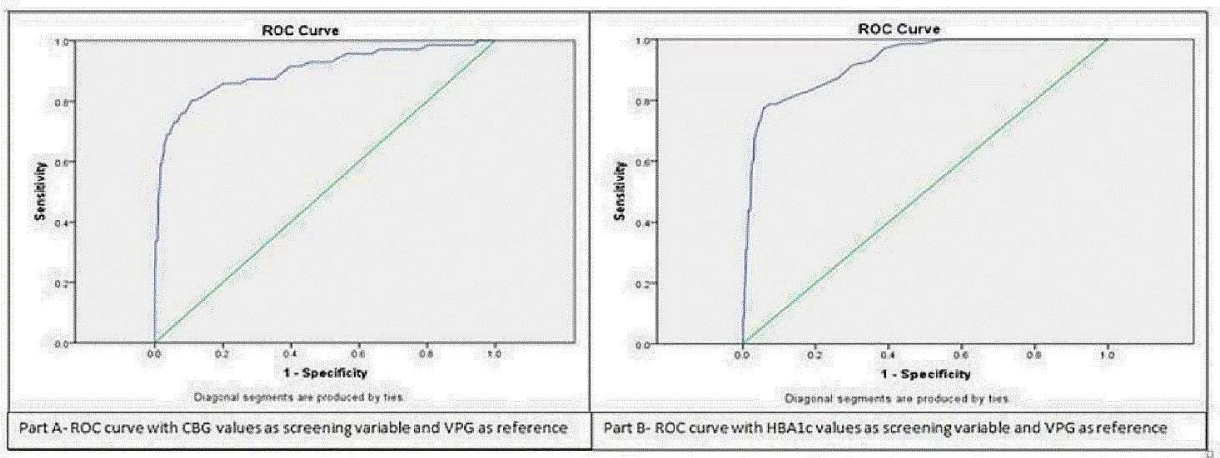

Figure 2. ROC curve with screening variables and VPG as reference.

Table 3. ROC curve indexes for $\mathrm{CBG}$ and $\mathrm{HbA1c}$ vs. VPG tests.

\begin{tabular}{lll}
\hline & CBG & HbA1c \\
\hline Cut point (as recommended) & $126(\mathrm{mg} / \mathrm{dl})$ & $6.5(\%)$ \\
\hline No. positive & 91 & 264 \\
\hline No. negative & 966 & 793 \\
\hline AUC & .0902 & 0.925 \\
\hline SE & 0.023 & 0.015 \\
\hline p-value & $<0.001$ & $<0.001$ \\
\hline $95 \% C l:$ & $0.856-0.948$ & $.0896-0.954$ \\
\hline Sensitivity & 80.30 & 77.50 \\
\hline Specificity & 89.10 & .9420 \\
\hline Optimal cut point & 116.50 & 7.15 \\
\hline
\end{tabular}


Table 4. Sensitivity, specificity, PPV and NPV for CBG and HbA1c vs. VPG based on cut points defined by ROC curve analysis.

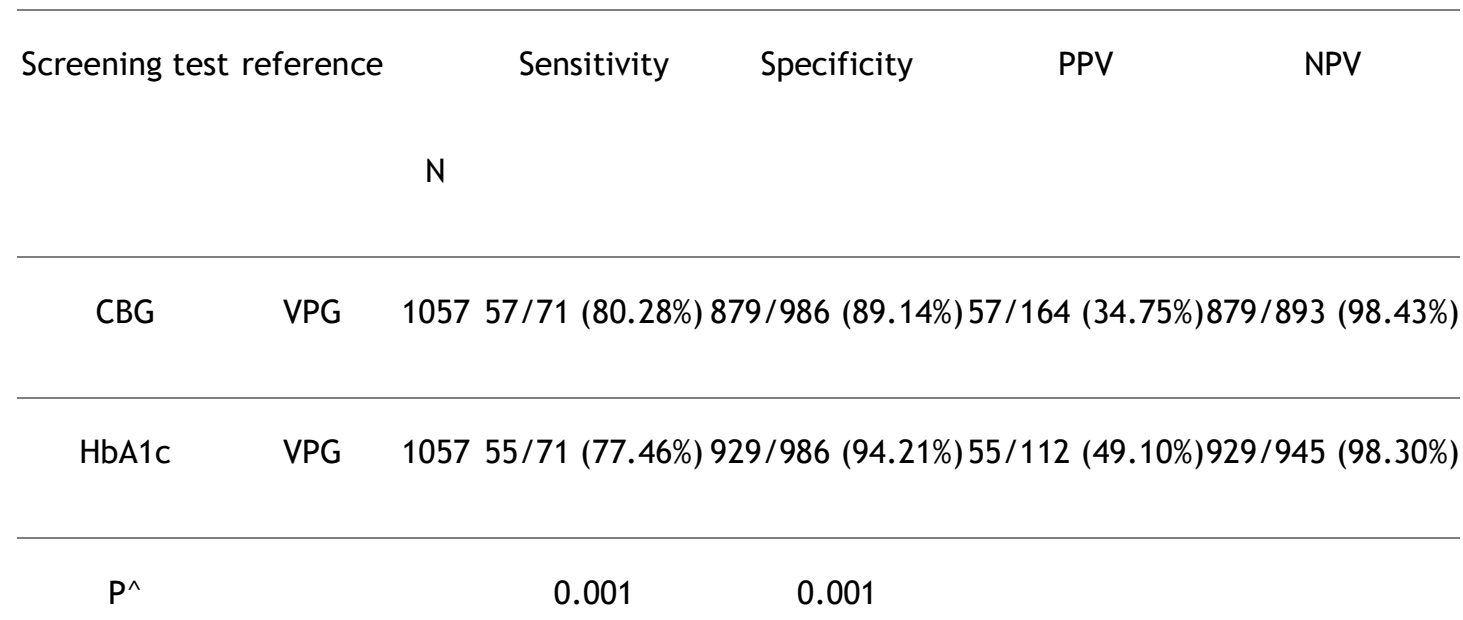

CBG $\geq 116.50(\mathrm{mg} / \mathrm{dl}) ; \mathrm{VPG} \geq 126(\mathrm{mg} / \mathrm{dl})$ and clinical diagnosis; PPV= Positive predictive value; NPV= Negative predictive

value; HbA1c $\geq 7.15$; ^compared with indexes based on WHO's recommendation cut points (CBG $\geq 126 \mathrm{mg} / \mathrm{dl} \& \mathrm{HbA} 1 \mathrm{c} \geq 6.5 \%$ ).

\section{Discussion:}

Early detection of DM is essential in the prevention and management of the related lifethreatening complications. An efficient screening program for early diagnosis of DM is essential to prevent the DM complications. Although several tests are introduced to identify diabetic patients, serious debates are still going over the validity and reliability of the results (21-23). The inconsistency in the validity of test results is due to several reasons. For example, in a study conducted in India, a bimodal distribution of fasting blood glucose was observed. Obviously, this phenomenon could not be detected by FBS with a cut-point between 140-120 mg/dl. (24) In addition, the American Diabetes Association (ADA) has suggested that glucose level of blood changes over time depending on several factors including the disease progress (22). In fact, despite the presence of DM, the metabolic changes maybe not big enough to detectably raise blood sugar (25). CBG is a test that is more frequently used due to its low cost and ease of use in DM screening programs. However, in several studies, the validity of the results of CBG test has been questioned $(23,26)$. Technical issues as well as environmental, psychological and medical conditions are listed as factors that affect the validity of the results of a test run by a glucometer (23).

In this study, sensitivity, specificity and positive and negative predictive values of the two DM diagnostic (selecting VBG as a gold standard test) were almost similar for CBG $(69.01 \%, 95.74 \%$, $53.84 \%$ and $97.72 \%$ reported by the current study respectively) but significantly lower for $\mathrm{HbA1c}$ $(84.50 \%, 79.31 \%, 22.72 \%$ and $98.61 \%$ reported by the current study respectively) when compared to what reported by Benja Muktabhant et al, (27). According to the report, the sensitivity, specificity, and positive and negative predictive values for CBG and $\mathrm{HbA} 1 \mathrm{c}$ were $81.4 \%, 97.8 \%, 71.4 \%$ and $98.7 \%$, and $39.70 \%, 96.70 \%, 56.80 \%$ and $93.70 \%$ respectively. However, another study on pregnant women by Balaji Bhavadharini et al. provided different results (28). Accordingly, the sensitivity, specificity, positive and negative predictive values for CBG test were $70.80 \%, 63.00 \%, 18.00 \%$ and $95.00 \%$ respectively that are in accordance with sensitivity and negative predictive value of the current study. It is to be noted that sensitivity and specificity are independent of the prevalence of the disease in a community, but the positive predictive value increases when prevalence increases and negative 
predictive value increases when the prevalence of the disease decreases. As a result, the predictive values of a test in a community are not comparable to those communities with different prevalence rates (29).

As recommended by American and European Diabetes Associations, HbA1c is commonly used as a test for screening or clinical diagnosis of diabetes $(8,30)$. Recently, the International Diabetes Federation and the World Health Organization also recommended $\mathrm{HbA} 1 \mathrm{c}$ test as the diagnostic test for diabetes $(8,30)$. In Iran, following WHO, HbA1c test is used for the diagnosis of diabetes with a cut-point at $6.50 \%$ (20). However, some studies indicated significant contradictions in the results of the test. For example, according to a report from the International Expert Committee for Diagnosis and Classification of Diabetes, HbA1c test results may be affected by conditions such as hemoglobinopathies, pregnancy, uremia, blood transfusion, and hemolytic anemia and also by the applied laboratory methods. However, based on the results of a study by D. M. Nathan et al., due to the strong correlation between $\mathrm{HbA} 1 \mathrm{c}$ test result and diabetic complications, the test is highly recommended as a DM care assessment measure (31). However, the use of HbA1c is more costly and requires sophisticated laboratory facilities which are hardly affordable by many developing countries. In addition, in a study, results of $\mathrm{HbA1c}$ compared to two-hour blood glucose levels and fasting blood glucose was less accurate in identifying people who were at risk of diabetes (32). The researchers used $\mathrm{HbA} 1 \mathrm{c}$ as a screening test and they announced sensitivity, specificity, and positive and negative predictive values for $\mathrm{HbA} 1 \mathrm{c}$ as $84.50 \%, 79.31 \%, 22.72 \%$ and $98.61 \%$ respectively. However, Farahani et al compared HbA1c results with VPG as the gold standard test (with a cutpoint at $110 \mathrm{dl} / \mathrm{mg}$ ) and obtained $100 \%$ sensitivity, $12.50 \%$ specificity, $82.10 \%$ positive and $66.70 \%$ negative predictive values (33), figures fundamentally different from what observed by the current study.

The current evaluation study on 1057 participants, who had no history of diabetes, measured the validity indexes of DSP in the Iranian population. Based on the results, when CBG (with a cut point at $126 \mathrm{mg} / \mathrm{dl}$ ) as a screening test and VPG (with a cut point at $126 \mathrm{mg} / \mathrm{dl}$ ) as a gold standard test were applied, sensitivity, specificity and positive predictive value of the tests appeared to be convincingly high when compared to the results from other countries. In addition, based on the results from ROC curve analysis, the recommended cut-point for CBG was calculated at $116.50 \mathrm{mg} / \mathrm{dl}$ with optimal sensitivity and specificity. When the performance of the NSPD is compared with the results of studies from European (AUC $=0.844$ ) and Arab (AUC=0.847) countries, the performance of $\mathrm{HbA1c}$ and CBG for the Iranian DSP is significantly better ( $\mathrm{AUC}=0.925$ for $\mathrm{HbA1c}$ and $\mathrm{AUC}=0.902$ for CBG)(18). However, using CBG test, $30.99 \%$ of people with DM were not detected while $4.26 \%$ of the healthy subjects undergone unnecessary clinical and laboratory procedures (false positive). When $\mathrm{HbA1c}$ was used as a screening test, $15.50 \%$ of diabetes patients were not detected while $20.69 \%$ of the healthy subjects undergone unnecessary clinical and laboratory procedures (false positive). In that regard, the best cut point for $\mathrm{HbA1c}$ was at $7.15 \%$, with sensitivity and specificity of $77.50 \%$ and $94.20 \%$ respectively.

Compared to several other countries, the performance of the Iranian SDP is reasonably better. This may be due to the differences in the defined criteria for selecting populations for screening (highrisk groups) in different countries. In this study, ROC analysis suggested new cut-points for even better performance of DSP in Iran. Further studies are needed to understand different aspects of the suggested cut points and the risk factors selected by DSP to define the high-risk population to achieve a better performance of the program.

\section{Implications for Policy \& Practice}

This study evaluated the performance of diabetes screening of Iran using a population-based sampling method

All procedures, instruments, and personnel used in this study were similar to what is used by the national screening program making the results more representative and applicable

New cut points are provided to increase the performance of the screening tests 
3 of 12

\section{Limitations:}

The participants in this study were all rural residences with different lifestyles of the urban population. As a result, our findings should be validated in urban populations.

\section{Acknowledgment:}

The present study is a part of M.Sc. thesis (Evaluation of National Iranian DM screening program) written by Fateme Kianpour under supervision of Dr. Mohamad Fararouei. The authors appreciate the help of health workers and the study participants for their cooperation. The present study was also supported by Gerash University of Medical Sciences, Gerash, Iran

\section{Decelerations:}

\section{Ethics approval and consent to participate:}

The participants were assured that their information is used for research proposes only. Because of the illiteracy of a significant number of parents, verbal consents were obtained from the parents. The study protocol was reviewed and approved by the ethical committee of Shiraz University of Medical Sciences.

\section{Consent for Publication:}

Not Applicable

\section{Availability of data and material:}

The datasets generated and/or analyzed during the current study are not publicly available due to its being the intellectual property of Shiraz University of Medical Sciences but are available from the corresponding author on reasonable request.

\section{Competing interests:}

The authors declare no conflict of interest.

\section{Funding:}

This study was conducted as a thesis for MSc degree in epidemiology and was financially supported by Shiraz University of Medical Sciences, Shiraz, Iran. (Grant number:1634).

\section{Authors' contributions:}

F.K and M.D were responsible for data collection and data analysis; M.M was responsible for coordination and management and manuscripts preparation; J.H was responsible for measurement methods and interpretation of results. M.F was responsible for coordination and management of the project and data analysis and preparation of the manuscript. All authors have read and approved the manuscript.

\section{Reference:}

1. Waine C. Osteoporosis: prevention and management in primary care. BMJ. 1997;314:1056 9.

2. Melmed S. Williams Textbook of Endocrinology. 13, editor: Elsevier; 2016. 
4 of 12

3. Baghaei A, Sarrafzadegan N, Rabiei K, Gharipour M, Tavasoli AA, Shirani S, et al. How effective are strategies for non-communicable disease prevention and control in a high risk population in a developing country? Isfahan Healthy Heart Programme. Archives of medical science : AMS. 2010;6(1):24-31.

4. Faramarzi H, Bagheri P, bahrampour A, halimi L. The Comparison of Prevalence of Diabete and Hypertension Between Rural Areas of Fars and Rural Area of EMRO Region. Iranian Journal of Endocrinology and Metabolism. 2011;13(2):157-64.

5. Salem Z, Neshat A, Bagherian K, Sheykh fatholahi M, Sajadi M. The prevalence of type II diabetes in the population over 30 years in Rafsanjan, 2000. Journal of Rafsanjan University of Medical Sciences and health services. 2004;3(1):9-16.

6. World Health Organization.glabal report on diabetes http://apps.who.int2016 [

7. NCD Risk Factor Collaboration.Worldwide trends in diabetes since 1980: a pooled analysis of 751 population-based studies with $4 \cdot 4$ million participants. The Lancet. 2016;387(10027):151330 .

8. Jia W. Standardising HbA1c-based diabetes diagnosis: opportunities and challenges. Expert Review of Molecular Diagnostics. 2016;16(3):343-55.

9. Limpaphayom KK, Taechakraichana N, Jaisamrarn U, Bunyavejchevin S, Chaikittisilpa S, Poshyachinda M, et al. Prevalence of osteopenia and osteoporosis in Thai women. Menopause. 2001;8(1):65 - 9 .

10. Brussels B. IDF Diabetes Atlas, in International Diabetes Federation, I.D. Federation, Editor. 2012 [updated 2012; cited], International Diabetes Federation: Available from: http://www.idf.org/diabetesatlas.

11. Gupta SK, Singh Z, Purty AJ, Mohan V. Diabetes prevalence and its risk factors in urban Pondicherry. International journal of diabetes in developing countries. 2009;29(4):166-9.

12. Gupta SK, Singh Z, Purty AJ, Kar M, Vedapriya D, Mahajan P, et al. Diabetes prevalence and its risk factors in rural area of Tamil Nadu. Indian journal of community medicine. 2010;35(3):396.

13. Seuring T, Archangelidi O, Suhrcke M. The Economic Costs of Type 2 Diabetes: A Global Systematic Review. Pharmacoeconomics. 2015;33(8):811-31.

14. Tunstall-Pedoe H. Preventing Chronic Diseases. A Vital Investment: WHO Global Report. Geneva: World Health Organization, 2005. pp 200. CHF 30.00. ISBN 924 1563001. Also published on http://www. who. int/chp/chronic_disease_report/en. International Journal of Epidemiology. 2006;35(4):1107-. 
5 of 12

15. Derakhshan A, Sardarinia M, Khalili D, Momenan AA, Azizi F, Hadaegh F. Sex specific incidence rates of type 2 diabetes and its risk factors over 9 years of follow-up: Tehran Lipid and Glucose Study. PloS one. 2014;9(7):e102563.

16. Peykari N. A comprehensive study on national and sub-national trends, burden, and inequality of fasting plasma glucose and diabetes prevalence in Iranian population (1990-2013). Epidemiological science, PhD [thesis]. 2015.

17. Zakerkish M, Rahimi N, Marefati H, Kovsarian Z. Evaluation of Cardiovascular Risk Factors in Diabetic Patients in Two Regions with Differences in Race, Culture and Climate. Jundishapur Scientific Medical Journal. 2013;12(4).

18. Bertran EA, Berlie HD, Taylor A, Divine G, Jaber LA. Diagnostic performance of HbA1c for diabetes in Arab vs. European populations: a systematic review and meta-analysis. Diabet Med. 2017;34(2):156-66.

19. Hajian-Tilaki K. Sample size estimation in diagnostic test studies of biomedical informatics. Journal of biomedical informatics. 2014;48:193-204.

20. Yavari P. Diabetes. Epidemiology Textbook of Prevalence Disease in Iran. 2. Tehran: gapnashr; 2014. p. 73-84.

21. Genuth S, Alberti KG, Bennett P, Buse J, Defronzo R, Kahn R, et al. Follow-up report on the diagnosis of diabetes mellitus. Diabetes Care. 2003;26(11):3160-7.

22. Sacks DB. A1C Versus Glucose Testing: A Comparison. Diabetes Care. 2011;34(2):518-23.

23. Tonyushkina K, Nichols JH. Glucose Meters: A Review of Technical Challenges to Obtaining Accurate Results. Journal of diabetes science and technology (Online). 2009;3(4):97180.

24. Rushforth NB, Miller M, Bennett PH. Fasting and two-hour post-load glucose levels for the diagnosis of diabetes. The relationship between glucose levels and complications of diabetes in the Pima Indians. Diabetologia. 1979;16(6):373-9.

25. American Diabetes Association.Diagnosis and classification of diabetes mellitus. Diabetes care. 2014;37(Supplement 1):S81-S90.

26. Parwaiz M, Lunt H, Florkowski CM, Logan FJ, Irons L, Perwick C, et al. Assessment of glucose meter performance at the antenatal diabetes clinic: exploration of patient-related and pre-analytical factors. Annals of clinical biochemistry. 2014;51(Pt 1):47-53.

27. Muktabhant B, Sanchaisuriya P, Sarakarn P, Tawityanon W, Trakulwong M, Worawat S, et al. Use of glucometer and fasting blood glucose as screening tools for diabetes mellitus type 2 and glycated haemoglobin as clinical reference in rural community primary care settings of a middle income country. BMC Public health. 2012;12(1):349. 
6 of 12

28. Bhavadharini B, Mahalakshmi MM, Maheswari K, Kalaiyarasi G, Anjana RM, Deepa M, et al. Use of capillary blood glucose for screening for gestational diabetes mellitus in resourceconstrained settings. Acta Diabetologica. 2016;53:91-7.

29. mausner j, kramer s. screening for diaease detection. In: janghorbani $m$, editor. an introductory text epidemiology. 1. kerman: Cultural Services Publishing; 2010. p. 321 - 54.

30. Report of a World Health Organisation Consultation:Use of glycated haemoglobin (HbA1c) in the diagnosis of diabetes mellitus. Diabetes Res Clin Pract. 2011;doi:10.1016/j.diabres.2011.03.012.

31. Nathan DM, Buse JB, Davidson MB, Ferrannini E, Holman RR, Sherwin R, et al. Medical management of hyperglycaemia in type 2 diabetes mellitus: a consensus algorithm for the initiation and adjustment of therapy: a consensus statement from the American Diabetes Association and the European Association for the Study of Diabetes. Diabetologia. 2009;52(1):1730.

32. Lorenzo C, Wagenknecht LE, Hanley AJG, Rewers MJ, Karter AJ, Haffner SM. A1C Between 5.7 and $6.4 \%$ as a Marker for Identifying Pre-Diabetes, Insulin Sensitivity and Secretion, and Cardiovascular Risk Factors. The Insulin Resistance Atherosclerosis Study (IRAS). 2010;33(9):2104-9.

33. Farahani H, Naeimi A. Comparison of glycosylated Hemoglobin and oral Glucose tolerance test in diagnosis of diabetes in person with impaired fasting Glucose. Arak Medical University Journal (Rahavard Danesh) 2004;7(29):33 -8. 